\title{
A two-fluid model describing the finite-collisionality, stationary Alfvén wave in anisotropic plasma
}

\author{
S. M. Finnegan ${ }^{1}$, M. E. Koepke ${ }^{1}$, and D. J. Knudsen ${ }^{2}$ \\ ${ }^{1}$ Department of Physics, West Virginia University, Morgantown, WV 26506-6315, USA \\ ${ }^{2}$ Department of Physics and Astronomy, University of Calgary, Calgary, Alberta, Canada
}

Received: 7 May 2008 - Revised: 13 October 2008 - Accepted: 13 October 2008 - Published: 9 December 2008

\begin{abstract}
The stationary inertial Alfvén (StIA) wave (Knudsen, 1996) was predicted for cold, collisionless plasma. The model was generalized (Finnegan et al., 2008) to include nonzero values of electron and ion collisional resistivity and thermal pressure. Here, the two-fluid model is further generalized to include anisotropic thermal pressure. A bounded range of values of parallel electron drift velocity is found that excludes periodic stationary Alfvén wave solutions. This exclusion region depends on the value of the local Alfvén speed $V_{A}$, plasma beta perpendicular to the magnetic field $\beta_{\perp}$ and electron temperature anisotropy.
\end{abstract}

\section{Introduction}

The existence of low-frequency (wave frequency $\omega$ much smaller than the ion cyclotron frequency $\omega_{\mathrm{ci}}$ ) magnetohydrodynamic waves with parallel wave phase speed $V_{A} \equiv B_{0} / \sqrt{\mu_{0} m_{\mathrm{i}} n}$ was first predicted by Hannes Alfvén in 1942 (Alfvén, 1942) ( $m_{\mathrm{i}}$ is the ion mass, $n$ is the plasma density and $B_{0}$ is the background magnetic field). For large wavelengths $\lambda_{\perp}$ perpendicular to $B_{0}$, the parallel electric field is insignificantly small and incapable of accelerating electrons producing current-induced perturbations to the background magnetic field. For the shear-mode Alfvén wave, ion polarization current in the direction perpendicular to $B_{0}$ is balanced by magnetic-field-aligned current (i.e., parallel) carried by electrons. When $\lambda_{\perp}$ becomes small, comparable to the electron inertial length $\lambda_{\mathrm{e}} \equiv c / \omega_{\mathrm{pe}}$ for the case when $\beta<m_{\mathrm{e}} / m_{\mathrm{i}}$ ( $\beta=$ kinetic pressure normalized to magnetic field pressure), or comparable to the ion acoustic gyroradius $\rho_{\mathrm{s}}=\sqrt{T_{e \|} / m_{i}} / \omega_{c i}$ in higher- $\beta$ plasma, the parallel electric

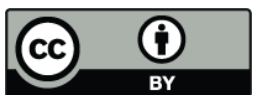

Correspondence to: S. M. Finnegan (sfinnega@mix.wvu.edu) field becomes significant and the wave becomes dispersive (Goertz and Boswell, 1979; Hasegawa and Uberoi, 1982). In this small- $\lambda_{\perp}$ limit, parallel electron acceleration can result in significant perturbations $\delta \mathbf{B}$ to the background magnetic field $\mathbf{B}_{0}$. Waves in the low- $\beta$ and high- $\beta$ limits are termed "inertial" and "kinetic", respectively, and together they are known as a dispersive Alfvén wave (e.g., Stasiewicz, 2005). The dispersive Alfvén wave has been studied extensively because of its possible role in accelerating electrons in the Earth's magnetosphere, particularly the auroral ionosphere, where the inertial wave can generate transient $(\sim 1 \mathrm{~s})$ bursts of electron energy at energies up to $\sim 1 \mathrm{keV}$ (e.g., Hui and Seyler, 1992; Kletzing, 1994; Lysak and Song, 2003; Chaston et al., 2004; Seyler and Liu, 2007). The dispersive Alfvén wave also leads to electron acceleration as a result of interhemispheric field-line resonances having periods of $\sim 10 \mathrm{~min}$ (e.g., Streltsov and Lotko, 1995; Rankin et al., 1999). See the comprehensive review by Stasiewicz et al. (2000). The linear dispersion relation for the dispersive Alfvén wave in both the inertial and kinetic limits has been verified in laboratory experiments (e.g., Allen et al., 1959; Jephcott, 1959; Wilcox et al., 1960; Jephcott and Stocker, 1962; Gekelman et al., 1997; Leneman et al., 1999; Vincena et al., 2004).

The dispersive Alfvén wave can be described in the context of two-fluid theory. Linear properties are derived by neglecting the nonlinear term $\mathbf{v} \cdot \nabla$ of the complete timederivative operator $(d / d t=\partial / \partial t+\mathbf{v} \cdot \nabla)$. Seyler and Wahlund (1995) predicted that inclusion of this nonlinearity leads to Alfvén wave steepening, wave breaking, and enhanced wave dissipation. Those authors also found a solution predicting a purely spatial perturbation in plasma density in the zerofrequency limit.

It is often convenient to study nonlinear waves in the reference frame in which the wave is stationary. In the wave-stationary frame, having velocity $\mathbf{V}_{f r}=\mathbf{V}_{p h}=(\omega / k) \hat{k}$, with respect to the lab frame, the wave does not fluctuate

Published by Copernicus Publications on behalf of the European Geosciences Union and the American Geophysical Union. 
in time so the total time derivative is $d / d t=-\mathbf{V}_{p h} \cdot \nabla$ and $\nabla \times \mathbf{E}=-\partial \mathbf{B} / \partial t=0$. A plasma species $j$ which is drifting in the lab-frame with velocity $\mathbf{V}_{j}$, will have a non-zero relative drift in the wave-frame and the total time derivative is $d / d t=\left(\mathbf{V}_{j}-\mathbf{V}_{p h}\right) \cdot \nabla$ in the wave frame. Previous studies of non-linear Alfvén waves made in the wave-frame (e.g., Hasegawa and Mima, 1976; Kotsarenko et al., 1998; Wu, 2003; Dubinin et al., 2005; Stasiewicz, 2005) have assumed that the background plasma is at rest in the lab-frame $\mathbf{V}_{j}=0$ so that $d / d t=-\mathbf{V}_{p h} \cdot \nabla$. Studies of the stationary Alfvén wave (StA) (e.g., Knudsen, 1996; Finnegan et al., 2008) however consider the wave to be stationary in the lab-frame $\mathbf{V}_{p h}=0$ and that the background plasma drift is non-zero $\mathbf{V}_{j} \neq 0$ in the lab frame so that $d / d t=\mathbf{V}_{j} \cdot \nabla$. Although both approaches describe solutions in the wave frame, each approach $\left(d / d t=\left(\mathbf{V}_{j}-\mathbf{V}_{p h}\right) \cdot \nabla\right.$ in the wave frame or $d / d t=\mathbf{V}_{j} \cdot \nabla$ in the lab frame) is associated with a different phenomenon in that the first case involves a fluctuating, traveling pattern and the second case involves a non-fluctuating, non-traveling pattern. In contrast to the dispersive Alfvén wave, the inclusion of the interaction between dc cross-field plasma flow and an initial dc field-aligned current provides a dc free energy source to support the non-fluctuating, nontraveling StA pattern in the absence of a structured driver. To summarize, whereas one approach is to choose either the wave frame or the lab frame (in which a Doppler shift is introduced), we treat the problem in a single frame of reference (the lab frame) within which the wave phase velocity is zero.

Whereas the seminal paper for dispersive Alfvén waves is Goertz and Boswell (1979), the seminal papers for stationary Alfvén waves are Mallinckrodt and Carlson (1978) and Maltsev et al. (1977). The term "stationary Alfvén wave" was introduced by Maltsev et al. (1977) to describe a stationary electromagnetic structure resulting from plasma convection past a conducting strip. The application of the term was broadened by Mallinckrodt and Carlson (1978) to include structuring from a moving field source in a magnetized plasma. The term "Alfvén wing" is used to describe a stationary wave resulting from moving conductors, for example in the case of Io orbiting within Jupiter's magnetosphere (Chust et al., 2005) or as a result of a conducting tether orbiting with a spacecraft (Sallago and Platzeck, 2004). In these cases, the structure of the stationary wave is imposed by the source, although Chust et al. (2005) argue that additional filamentation can result. In contrast to previous descriptions of Alfven wings, the nonlinear two-fluid model described here and by Knudsen (1996) includes the effects of electron inertia, leading to a self-consistent StA wave that restructures a large-scale current sheet into a new electromagnetic equilibrium, and does not require a structured source. Also, as with its time-varying counterpart - the dispersive Alfven wave the StA wave develops a significant parallel electric field as a result of finite electron mass and plasma temperature.

In this paper, we are concerned with the limit $d / d t=\left(\mathbf{V}_{j}\right) \cdot \nabla$. Knudsen (1996) predicted that in the presence of background, perpendicular, plasma convection through the wave fields, the $\mathbf{V}_{j} \cdot \nabla$ term alone generates Alfvén-wave-like behavior, with electromagnetic structures evolving in space as a result of the apparent time variation seen by plasma particles convecting across static (electric and magnetic) field structures. Within otherwise large-scale sheets of parallel current, the StA wave imposes structure both on plasma density and on parallel electron energy. The inclusion of electron and ion temperature anisotropies in the wave model is motivated by ionospheric observations (e.g., Oyama and Abe, 1987; Demars and Schunk, 1987) while the inclusion of collisions (which tend to reduce temperature anisotropy) is motivated by laboratory experiments, designed for verifying the existence of the stationary inertial Alfvén wave (StIA) (StA wave in the inertial $\beta<m_{e} / m_{i}$ limit) which are currently underway (Koepke et al., 2008). For completeness in developing intuition, anisotropy and collisions are evaluated separately and together.

\section{Two-fluid equations}

In this paper, we present a two-dimensional (Cartesian) wave model describing uniform plasma flow $V_{d}$, due to a convection electric field $E_{y}=V_{d} B_{0}$, across a magnetic-field-aligned current sheet $\left(\partial / \partial_{y}=0\right)$ within a warm, anisotropic, collisional plasma. Solutions are sought for Alfvénic, stationary wave patterns having wave vector $\mathbf{k}$ oriented nearly perpendicular to a background magnetic field $\left(\hat{z} B_{0}+\hat{y} B_{y 0}\right)$, with $B_{y 0} \ll B_{0}$. The equations are expressed appropriately for the lab-stationary, wave-stationary frame of reference $\left(\partial / \partial_{t}=0\right.$, $V_{f r}=0$ ). Wave equations describing an StA wave are derived from the following set of anisotropic, time-independent, twofluid equations,

$$
\begin{aligned}
& \left(\mathbf{V}_{j} \cdot \nabla\right) \mathbf{V}_{j}=\frac{q_{j}}{m_{j}}\left[\mathbf{E}+\left(\mathbf{V}_{j} \times \mathbf{B}\right)\right]-\frac{\nabla \cdot \mathbf{P}_{j}}{m_{j} n_{j}}+\frac{\mathbf{R}_{j}}{m_{j} n_{j}}, \\
& \nabla \cdot\left(n_{j} \mathbf{V}_{j}\right)=0, \\
& \nabla \times \mathbf{B}=\mu_{0} \mathbf{J} . \\
& \nabla \times \mathbf{E}=0 \\
& \nabla \cdot \mathbf{B}=0 .
\end{aligned}
$$

Here, $j$ denotes particle species, $q_{j}$ is the particle charge, $\mathbf{V}_{j}$ is the fluid velocity, $n_{j}$ is the particle density, $\mathbf{P}_{j}$ is the anisotropic pressure tensor, and $\mathbf{R}_{j}$ is the collisional resistivity. The set of Eqs. (1-5) are closed through the quasineutrality condition $n_{e} \approx n_{i}=n$.

The electron fluid velocity is comprised of the initial electron drifts across the magnetic field and along the magnetic field $\mathbf{V}_{e 0}=\hat{x} V_{d}+\hat{z} V_{e z 0}$, and the wave modified velocity $\mathbf{v}_{e}$

$\mathbf{V}_{e}=\mathbf{V}_{e 0}+\mathbf{v}_{e}$. 
Similarly, the ion fluid velocity is comprised of the initial ion drift across the magnetic field $\mathbf{V}_{i 0}=\hat{x} V_{\mathrm{i} \times 0}$ and the wave modified velocity $\mathbf{v}_{i}$

$\mathbf{V}_{i}=\mathbf{V}_{i 0}+\mathbf{v}_{i}$

The initial ion drift across the magnetic field is the sum of the $\mathbf{E} \times \mathbf{B}$ drift $V_{d}$ and an initial polarization drift $V_{\text {ip0 }}$ i.e. $V_{\mathrm{i} \times 0}=V_{d}+V_{\mathrm{ip} 0}$. The initial polarization drift of the electrons is neglected since it is a factor of $m_{e} / m_{i}$ smaller than that of the ions.

The electron and ion collisional resistivity terms are,

$\mathbf{R}_{i}=q_{e} N \eta_{\| 0} \mathbf{J}_{\|}+q_{e} N \eta_{\perp 0} \mathbf{J}_{\perp}-n m_{i} v_{i n} \mathbf{V}_{i}$,

and

$\mathbf{R}_{e}=-q_{e} N \eta_{\| 0} \mathbf{J}_{\|}-q_{e} N \eta_{\perp 0} \mathbf{J}_{\perp}$,

where $\eta_{\| 0}$ is the initial parallel resistivity, $\eta_{\perp 0}$ is the initial perpendicular resistivity, $N$ is the initial background plasma density and $v_{i n}$ is the ion-neutral collision frequency. The velocity of neutrals is assumed to be negligible with respect to the fluid velocity of the ions i.e. $\mathbf{V}_{i}-\mathbf{V}_{N} \approx \mathbf{V}_{i}$. The parallel and perpendicular resistivites result from Coulomb interactions between the ions and electrons e.g. $\eta_{\| 0}=\left(m_{e} / q_{e}^{2} N\right) v_{e i \|}$ and $\eta_{\perp 0}=\left(m_{e} / q_{e}^{2} N\right) v_{e i \perp}$.

The anisotropic pressure tensor is given by

$\mathbf{P}_{j}=p_{j \perp} \mathbf{I}+\left(p_{j \|}-p_{j \perp}\right) \hat{b} \hat{b}=p_{j \perp}\left(\mathbf{I}-a_{j} \hat{b} \hat{b}\right)$.

Here $\hat{b}$ is the magnetic field-aligned unit vector, $\mathbf{I}$ is the unit tensor and $a_{j}$ represents the pressure anisotropy. We assume that the parallel and perpendicular pressures are $p_{j \|}=n T_{j \|}$ and $p_{j \perp}=n T_{j \perp}$ respectively ( $T_{j}$ is in energy units $\mathrm{eV}$ ). In this study we assume that both the parallel and perpendicular temperatures are constant, and thus the pressure anisotropy parameter $a_{j}$ is constant

$a_{j} \equiv 1-T_{j \|} / T_{j \perp}$

A graphical representation of the assumed Cartesian geometry, including vector directions of background magnetic field $B_{0}$ and initial particle drifts, for our two-dimensional StA wave model is shown in Fig. 1. The total magnetic field is composed of an assumed uniform background magnetic field in the z-direction $B_{0}$, the magnetic field structure associated with the background current channel $B_{y 0}$ and the wavefield $\delta \mathbf{B}\left(\mathbf{B}=\hat{y}\left(B_{y 0}+\delta B_{y}\right)+\hat{z}\left(B_{0}+\delta B_{z}\right)\right)$. The y-component of the wave-field $\delta B_{y}$ is not necessarily small compared to the y-component of the background field $B_{y 0}$. However, the y-component of the total field $B_{y}$ is assumed to be much smaller than the z-component of the background field $B_{0}$ i.e. $B_{y} / B_{0} \ll 1$, leading to the assumption that the angle between the magnetic field direction $\hat{b}$ and the $\mathrm{z}$-axis is small i.e. $\alpha \ll 1$. Only terms to first order in $B_{y} / B_{0}$ are retained. Terms of order $\delta B_{z} / B_{0}$ can be shown to be of order $B_{y}^{2} / B_{0}^{2}$

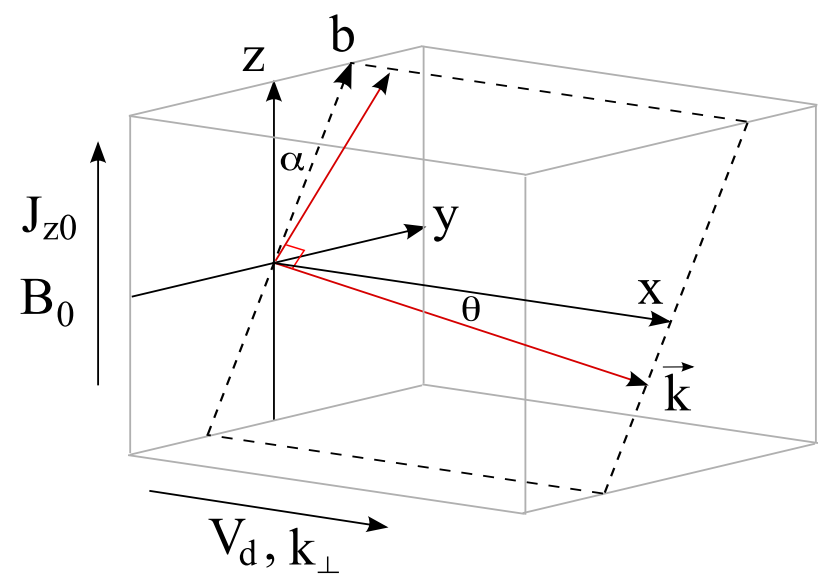

Fig. 1. Depiction of relevant vectors in the Cartesian geometry of our stationary Alfvén wave model.

and are subsequently neglected (e.g., Knudsen, 1996). The unit vector of the magnetic field-aligned direction is

$\hat{b} \equiv \frac{\mathbf{B}}{|\mathbf{B}|} \approx \hat{y} \frac{B_{y}}{B_{0}}+\hat{z}+O\left(\frac{\delta B_{z}}{B_{0}}\right)+O\left(\frac{B_{y}^{2}}{B_{0}^{2}}\right)$.

Solutions are sought for wave structures oriented at an oblique angle $\theta$ to the $\mathrm{x}$-axis (in the $\mathrm{b}$ - $\mathrm{x}$ plane). Thus the differential operators $\partial / \partial x$ and $\partial / \partial z$ are related by,

$\frac{\partial}{\partial z}=-\tan \theta \frac{\partial}{\partial x}$.

\subsection{Continuity relations}

The electron and ion continuity Eq. (2) are reduced to one dimensional differential equations in $x$ using Eq. (11), and integrated over the interval $\left[x_{o}, x\right]$ yielding expressions for the total particle density $n$ in terms of both the initial particle density $N=n\left(x=x_{0}\right)$ and the components of the particle fluid velocities

$\frac{n}{N}=\frac{1}{u_{i}-a}=\frac{U_{e}}{u_{e}}$.

Normalized functions of the electron and ion fluid velocities have been defined as $u_{e} \equiv 1-\left(v_{e z}+V_{e z 0}\right) / V_{\mathrm{phs}}$ and $u_{i} \equiv 1+v_{i x} / V_{\mathrm{i} \times 0}$, respectively with $V_{\mathrm{phs}}=V_{d} / \tan \theta$ being the effective parallel wave phase speed. Also, $U_{e} \equiv 1-V_{\mathrm{ez} 0} / V_{\mathrm{phs}}$ is the initial condition at $x=x_{0}$ for the function of electron velocity $\left(u_{e}\left(x=x_{0}\right)=U_{e}\right)$. In this study, we assume that the initial ion drift in $x$ is dominated by $\mathbf{E} \times \mathbf{B}$ motion, and that $v_{i z} \ll V_{A}$. With these assumptions, the quantity $a \equiv \tan \theta v_{i z} / V_{\mathrm{i} \times 0}$ can be neglected with respect to $u_{i}$ in Eq. (12). Particle densities are necessarily positive for physically meaningful solutions, thus only solutions for which both $U_{e} / u_{e}>0$, and $u_{i}>0$ are considered. 


\subsection{Parallel and perpendicular current density}

The field-aligned current density is assumed to be carried by the electrons and is given by

$J_{\|} \equiv \hat{b} \cdot \mathbf{J}=J_{\|} \approx q_{e} n_{e} V_{e z}$

Using Eq. (11), the parallel current $J_{\|}$is related to the perpendicular current $J_{\perp} \approx J_{x}$ through the condition that the current be divergence free, i.e., $\nabla \cdot \mathbf{J}=0$,

$J_{x}=-q_{e} N V_{d}\left(\frac{V_{\mathrm{i} \times 0}}{V_{d}}-\frac{1}{u_{i}}\right)$.

The perpendicular current results from the polarization drift of ions through the spatially inhomogeneous transverse electric field.

\subsection{Ion polarization drift}

The stationary analog of the ion polarization drift is calculated from the ion momentum Eq. (1).

$$
\begin{aligned}
\left(\frac{\Xi^{2}}{\omega_{c i}^{2}}+1\right) V_{i x}= & \frac{\Xi}{\omega_{c i} B_{0}}\left[E_{x}-\frac{T_{i}}{q_{i} n} \frac{\partial n}{\partial x}-\frac{N}{n} \eta_{\perp 0} J_{x}\right] \\
& +V_{d}-\frac{B_{y}}{B_{0}^{2}} E_{\|}+a_{i} \frac{V_{T i}^{2}}{V_{A}^{2}} \frac{J_{x}}{q_{i} n} \\
& +\frac{B_{y}}{B_{0}}\left(1-2 a_{i}\right) \frac{T_{i \perp}}{q_{i} B_{0}} \frac{1}{n} \frac{\partial n}{\partial z}
\end{aligned}
$$

Here the differential operator $\Xi$ is defined as $\Xi \equiv\left(\mathbf{V}_{i} \cdot \nabla\right)+v_{i n}, \omega_{c i}=q_{i} B_{0} / m_{i}$ is the ion gyrofrequency, and $V_{T i}=\left(T_{i \perp} / m_{i}\right)^{1 / 2}$ is the ion thermal speed. The definitions of the functions of electron and ion fluid velocity, along with Eq. (12) and the quasi-neutrality condition are used to solve Eq. (15) for the perpendicular variation of $E_{x}$.

$$
\begin{aligned}
\lambda_{e} & \frac{\partial \bar{E}_{x}}{\partial x}=\left(1+\frac{\bar{v}_{i n}^{2}}{\bar{\omega}_{c i}^{2}}\right)-\frac{p U_{e}}{u_{e}}\left(1+\bar{v}_{i n} \bar{E}_{x}\right) \\
+ & \bar{v}_{i n}\left[\frac{2}{\bar{\omega}_{c i}^{2} p U_{e}}-\beta_{m \perp}\left(\frac{\tau}{\tau+1}\right) \frac{p U_{e}}{u_{e}^{2}}\right] \lambda_{e} \frac{\partial u_{e}}{\partial x} \\
+ & {\left[\frac{1}{\bar{\omega}_{c i}^{2} p^{2} U_{e}^{2}}+\beta_{m \perp}\left(\frac{\tau}{\tau+1}\right) \frac{1}{u_{e}^{2}}\right] \lambda_{e}^{2}\left(\frac{\partial u_{e}}{\partial x}\right)^{2} } \\
+ & {\left[\frac{1}{\bar{\omega}_{c i}^{2} p^{2} U_{e}^{2}}-\beta_{m \perp}\left(\frac{\tau}{\tau+1}\right) \frac{1}{u_{e}^{2}}\right] u_{e} \lambda_{e}^{2} \frac{\partial^{2} u_{e}}{\partial x^{2}} . }
\end{aligned}
$$

Here, $\quad \bar{E}_{x} \equiv E_{x} /\left(\lambda_{e} \omega_{c i} B_{0}\right), \quad \bar{\omega}_{c i} \equiv \omega_{c i} /\left(V_{d} / \lambda_{e}\right)$, $\bar{v}_{i n} \equiv v_{i n} /\left(V_{d} / \lambda_{e}\right), \quad \tau \equiv T_{i \perp} / T_{e \perp}, \quad$ and $\quad \beta_{m \perp} \equiv \beta_{\perp}\left(m_{i} / m_{e}\right)$ where $\beta_{\perp} \equiv n\left(T_{e \perp}+T_{i \perp}\right) /\left(B_{0}^{2} / \mu_{0}\right)$. The factor $p$ is the ion polarization drift variable and is defined as

$p \equiv\left(1+V_{i p 0} / V_{d}\right)^{-1}=V_{d} / V_{\mathrm{i} \times 0}$.
We see in Eq. (16) that the ion polarization drift, and hence the ion polarization current, is unchanged by ion temperature anisotropy to first order in $B_{y} / B_{0}$.

\subsection{Parallel electric field}

The parallel component of the electric field is modified by the plasma $\mathbf{E} \times \mathbf{B}$ flow,

$E_{\|} \equiv \hat{b} \cdot \mathbf{E} \approx E_{z}+V_{d} B_{y}$.

This modification is of order $B_{y} / B_{0}$ and is retained. For nearly perpendicular, i.e. $k_{x} \gg k_{z}$, StA wave patterns, we assume that the balancing of the parallel component of electric field is governed primarily by the parallel electron dynamics. Thus, the parallel component of the electric field is calculated from the parallel component of the electron momentum equation.

$E_{\|}=\frac{m_{e}}{q_{e}}\left(\mathbf{V}_{e} \cdot \nabla\right) V_{e z}+\left(1-a_{e}\right) \frac{T_{e \perp}}{q_{e} n} \frac{\partial n}{\partial z}+\frac{N}{n} \eta_{\| 0} J_{\|}$.

Equation (19) reduces to a one-dimensional partial differential equation in $x$ using Eq. (11)

$$
\begin{aligned}
E_{\|}= & \frac{N}{n} \eta_{\| 0} J_{\|}+\frac{m_{e} \tan \theta}{q_{e}}\left(V_{\mathrm{phs}}-V_{e z}\right) \frac{\partial}{\partial x} V_{e z} \\
& -\frac{m_{e} \tan \theta}{q_{e}} \frac{\left(1-a_{e}\right) V_{T e}^{2}}{\left(V_{\mathrm{phs}}-V_{e z}\right)} \frac{\partial}{\partial x} V_{e z} .
\end{aligned}
$$

Here, $V_{T e}=\left(T_{e \perp} / m_{e}\right)^{1 / 2}$ is the electron thermal speed. We see in Eq. (20) that the parallel component of electric field, is balanced by parallel resistivity (first term on the right hand side), electron inertia (second term), and parallel electron pressure (last term). The $180^{\circ}$ phase difference between the inertial and thermal pressure terms was first identified by Goertz and Boswell (1979). Also, the portion of the parallel electric field balanced by finite electron conductivity is out of phase with the terms related to electron inertia and parallel electron thermal pressure. This phase difference plays a fundamental role in the spatial decay and growth of StA waves (Finnegan et al., 2008).

\subsection{Wave equations}

Differentiating Eq. (18) with respect to $x$ and combining Eqs. (3), (4), (10), (12), (13), (20), and the quasi-neutrality condition, yields a second-order, nonlinear, differential equation for the function $u_{e}$,

$$
\begin{aligned}
0= & \frac{\lambda_{e}^{2}}{2} \frac{\partial^{2} u_{e}^{2}}{\partial x^{2}}-\frac{V_{A}^{2}}{V_{\mathrm{phs}}^{2}}\left(1-a_{e}\right) \beta_{m \perp}\left(\frac{1}{\tau+1}\right) \lambda_{e}^{2} \frac{\partial^{2}}{\partial x^{2}} \ln u_{e} \\
& +\frac{V_{A}^{2}}{V_{\mathrm{phs}}^{2}} \frac{\partial \bar{E}_{x}}{\partial x}+\bar{v}_{e i \|} \lambda_{e} \frac{\partial u_{e}}{\partial x}+U_{e}\left(\frac{1}{u_{e}}-1\right) .
\end{aligned}
$$


Here, $\bar{v}_{e i \|}=\left(\lambda_{e} / V_{d}\right) \nu_{e i \|}$ is the normalized parallel electron collision frequency and substitution of Eq. (16) into Eq. (21) yields the wave equation for a resistive stationary Alfvén wave,

$$
\begin{aligned}
0= & \frac{\lambda_{e}^{2}}{2} \frac{\partial^{2} u_{e}^{2}}{\partial x^{2}}-\frac{V_{A}^{2}}{V_{\mathrm{phs}}^{2}}\left(1-\frac{a_{e}}{1+\tau}\right) \beta_{m \perp} \lambda_{e}^{2} \frac{\partial^{2}}{\partial x^{2}} \ln \left(u_{e}\right) \\
& +\bar{v}_{i n} \frac{V_{A}^{2}}{V_{\mathrm{phs}}^{2}}\left[\frac{2}{\bar{\omega}_{c i}^{2} p U_{e}}-\beta_{m \perp}\left(\frac{\tau}{\tau+1}\right) \frac{p U_{e}}{u_{e}^{2}}\right] \lambda_{e} \frac{\partial u_{e}}{\partial x} \\
& +\frac{V_{A}^{2} / V_{\mathrm{phs}}^{2}}{\bar{\omega}_{c i}^{2} p^{2} U_{e}^{2}} \frac{\lambda_{e}^{2}}{2} \frac{\partial^{2} u_{e}^{2}}{\partial x^{2}}-\left[p \frac{V_{A}^{2}}{V_{\mathrm{phs}}^{2}}\left(1+\bar{v}_{i n} \bar{E}_{x}\right)-1\right] \frac{U_{e}}{u_{e}} \\
& +\bar{v}_{e i \|} \lambda_{e} \frac{\partial u_{e}}{\partial x}-\left[U_{e}-\frac{V_{A}^{2}}{V_{\mathrm{phs}}^{2}}\left(1+\frac{\bar{v}_{i n}^{2}}{\bar{\omega}_{c i}^{2}}\right)\right] .
\end{aligned}
$$

Equation (22) is coupled to Eq. (16) through the polarization field $E_{x}$ and, together, they represent the closed set of equations describing StA wave modification of field-aligned electron drift. It is important to note that Eqs. (22) and (16) contain the full ion and electron convective nonlinearities $\left(\mathbf{V}_{j} \cdot \nabla\right)$ and are valid to first order in the magnetic perturbation $B_{y} / B_{0}$. The time scale on which collisions and cyclotron effects become important is identified through the normalization of the collision and cyclotron frequencies, respectively. In particular, these time-periods are normalized to the time required for the bulk plasma to drift $V_{d}(\mathbf{E} \times \mathbf{B}$ drift $)$ the distance of one electron inertial length, $\lambda_{e} / V_{d}$. Hence, particle collisions (cyclotron effects) become increasingly important as the number of collisions (gyro-periods) a particle undergoes as it transits across one perpendicular wave length of an StA wave structure increases (decreases).

Figure 2 shows numerical solutions to the coupled set of Eqs. (22) and (16) for the parallel electron velocity $V_{e s} / V_{\mathrm{es} 0}$, and plasma density $n / N$ for $V_{\mathrm{ez} 0}=-0.5 V_{A}$ and $V_{\mathrm{phs}}=0.2$ as a function of $x / \lambda_{e}$. The stationary inertial Alfvén wave structure in Fig. 2 is periodic with a wavelength of $\lambda_{0}=5.31 \lambda_{e}$. For the anti-parallel electron drift (APED) case shown, the StIA wave accelerates electrons in the direction of their initial drift and depletes the background plasma density. Knudsen (1996) and Finnegan et al. (2008) characterize the general properties of the StIA and the stationary kinetic Alfvén (StKA) wave in more detail.

\section{StA wave pattern formation}

Spatial StA wave patterns are supported by either a background magnetic-field-aligned current carried by electrons $J_{z 0}=q_{\mathrm{e}} n \mathrm{~V}_{\mathrm{ez} 0}$ or an initial perpendicular current carried by the ions $p \neq 1$. To show that periodic StA wave patterns do not form in the absence of both initial currents i.e. $V_{\mathrm{ez} 0}=0$

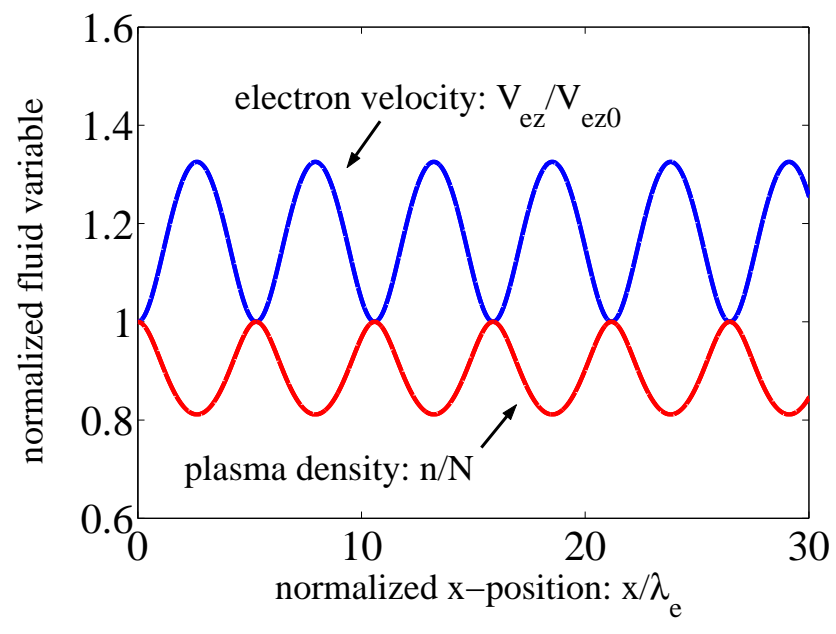

Fig. 2. Parallel electron velocity $V_{e s} / V_{\text {es } 0}$, and plasma density $n / N$ are plotted for $V_{\mathrm{ez} 0}=-0.5 V_{A}$ and $V_{\mathrm{phs}}=0.2$, as a function of $x / \lambda_{e}$. The StIA wave with initial anti-parallel electron drift (APED) accelerates electrons in the direction of their initial drift and depletes the background plasma density. This StIA wave structure has a wavelength of $\lambda_{0}=5.31 \lambda_{e} .\left(v_{i n}, v_{e \|}, v_{e \perp}, \tau, a_{e}, \beta_{m \perp}=0, \omega_{c i} \rightarrow \infty\right)$

and $p=1$ (see Fig. 3), we write Eq. (22) in a generalized form assuming that $v_{i n}=0$,

$f\left(v_{e z}\right) \frac{\partial^{2} v_{e z}}{\partial x^{2}}+g\left(v_{e z}, \frac{\partial v_{e z}}{\partial x}\right) \frac{\partial v_{e z}}{\partial x}+k_{x 0}^{2} v_{e z}=F$.

The driving term on the right hand side of Eq. (23) is

$$
\begin{aligned}
F=V_{A} & \left(1-\frac{V_{e z 0}}{V_{d}} \tan \theta\right)\left[\frac{V_{e z 0}}{V_{A}}+(1-p) \frac{V_{A}}{V_{d}} \tan \theta\right] \\
\times & {\left[\Lambda_{e}^{2} U_{e}^{2}-\frac{V_{A}^{2}}{V_{\mathrm{phs}}^{2}} \rho_{s}^{2}\left(1+\frac{V_{T i}^{2}}{C_{S}^{2}}\right)\right]^{-1}, }
\end{aligned}
$$

where $\quad \Lambda_{e}^{2} \equiv \lambda_{e}^{2}\left[1+\left(V_{A}^{2} / V_{\mathrm{phs}}^{2}\right)\left(\bar{\omega}_{c i}^{2} p^{2} U_{e}^{2}\right)^{-1}\right] \quad$ and $\rho_{s}=C_{S} / \omega_{c i}$ is the acoustic gyroradius, with $C_{S}=\sqrt{T_{e \|} / m_{i}}$ being the sound speed (e.g., Lysak and Lotko, 1996; Lysak and Song, 2003). Here, we see that the driver is spatially and temporally constant and depends on both the initial parallel current in the form of $V_{\mathrm{ez} 0}$ and initial perpendicular current represented by the polarization parameter $p$. For $p=1$, i.e. no initial perpendicular current, the StA wave is driven by the initial parallel current. When the initial parallel current is turned off i.e. $V_{\mathrm{ez} 0}=0$ the driving term is zero and without an initial perturbation, the StA wave pattern will not form. Thus, in the absence of both an initial parallel current and initial perpendicular current, i.e., $F=0$, a spatial StA wave pattern will not form.

In warm plasma $(\beta \neq 0)$, the formation of periodic StA wave patterns not only requires the initial currents discussed above but also requires that the parallel electron velocity 


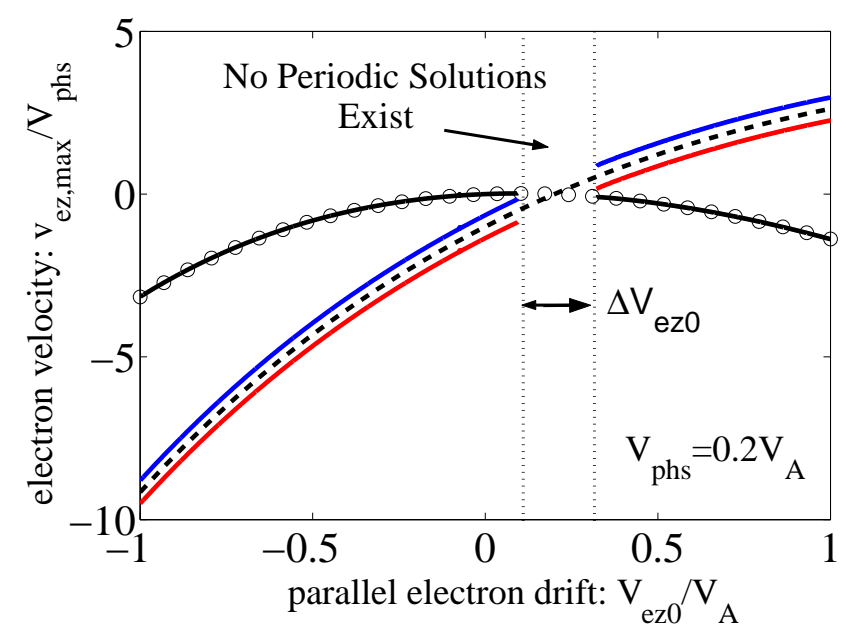

Fig. 3. The maximum deviation from the initial value in electron velocity $\left(v_{e s, \max } / V_{\mathrm{phs}}\right)$ is plotted as a function of the initial electron drift $V_{\mathrm{ez} 0}$ for $V_{\mathrm{phs}}=0.2 V_{A}$ with $\beta_{m \perp}=0.2$ and $\tau=1$. A region in $V_{\text {ez0 }}$ parameter-space exists for $\beta_{m \perp} \neq 0$ in which the StIA wave does not form. $\left(v_{i n}, v_{e \|}, v_{e \perp}=0\right.$, and $\left.\omega_{c i} \rightarrow \infty\right)$

$V_{e z}$ not pass through the effective wave phase speed $V_{\mathrm{phs}}$. That is to say that the parallel electron velocity must satisfy $V_{e z}<V_{\mathrm{phs}}$ for $V_{\mathrm{ez} 0}<V_{\mathrm{phs}}$ and $V_{e z}>V_{\mathrm{phs}}$ for $V_{\mathrm{ez} 0}>V_{\mathrm{phs}}$. When $V_{e z}$ passes through $V_{\text {phs }}$, the electrons are resonantly accelerated (e.g., Temerin et al., 1986; Kletzing, 1994) and the parallel component of the electric field becomes singular, a condition for which periodic solutions do not exist. To illustrate this we consider the collisionless $\left(\eta_{\| 0}=0\right)$ form of Eq. (20)

$E_{\|}=\frac{m_{e}}{q_{e}}\left[\left(V_{\mathrm{phs}}-V_{e z}\right)-\frac{\left(1-a_{e}\right) V_{T e}^{2}}{\left(V_{\mathrm{phs}}-V_{e z}\right)}\right] \frac{\partial}{\partial z} V_{e z}$.

When the effects of the parallel electron thermal pressure balance the effects of the electron inertia for $\partial V_{e z} / \partial z \neq 0$, the parallel component of electric field changes direction.

$0=\left(V_{\mathrm{phs}}-V_{e z}\right)-\frac{\left(1-a_{e}\right) V_{T e}^{2}}{\left(V_{\mathrm{phs}}-V_{e z}\right)}$.

This change in direction causes runaway acceleration of the electrons until $V_{e z}=V_{\mathrm{phs}}$ and the parallel electric field becomes singular. Solving Eq. (26) for $V_{e z}$, we obtain an expression for the amplitude limit for periodic StA wave patterns.

$\frac{V_{e z}^{-,+}}{V_{A}}=\frac{V_{\mathrm{phs}}}{V_{A}} \mp\left[\frac{\beta_{m \perp}\left(1-a_{e}\right)}{1+\tau}\right]^{1 / 2}$.

When the electrons are accelerated to $V_{e z}^{-} / V_{A}$ (for $V_{\mathrm{phs}}>V_{\mathrm{ez} 0}$ ) or $V_{e z}^{+} / V_{A}$ (for $V_{\mathrm{phs}}<V_{\mathrm{ez} 0}$ ), the parallel component of the electric field changes direction and periodic wave patterns do not form (see Fig. 3). In Fig. 3, numerical solutions to Eq. (22) for the maximum deviation from the initial value in electron velocity $\left(v_{\mathrm{es}, \max } / V_{\mathrm{phs}}\right)$ is plotted as a function of the initial electron drift $V_{\mathrm{ez} 0}$ for $V_{\mathrm{phs}}=0.2 V_{A}$ with $\beta_{m \perp}=0.2$ and $\tau=1 \quad\left(v_{i n}, v_{e \|}, v_{e \perp}=0\right.$, and $\left.\omega_{c i} \rightarrow \infty\right)$. The blue and red solid lines in Fig. (3) are $v_{e z, \max }-v_{e z}^{-}$ and $v_{e z, \max }-v_{e z}^{+}$, respectively. As $V_{\mathrm{ez} 0}$ approaches $V_{\mathrm{phs}}$ from the left, $v_{e z, \max } \rightarrow v_{e z}^{-}$and periodic wave patterns do not form (solid black line, $a_{e}=0$ ). Similarly, as $V_{\mathrm{ez} 0}$ approaches $V_{\mathrm{phs}}$ from the right, $v_{e z, \max } \rightarrow v_{e z}^{+}$and likewise periodic wave patterns do not form. When the parallel electron pressure is zero i.e. $a_{e}=1$ (solution shown with circles in Fig. 3), $V_{e z} \neq V_{\mathrm{phs}}$ everywhere in $V_{\mathrm{ez} 0}$ parameter-space except $V_{\mathrm{phs}}=V_{\mathrm{ez} 0}$, where the wave amplitude is also zero. The dashed line in Fig. 3 represents $v_{e z, \max }-\left(V_{\mathrm{phs}}-V_{\mathrm{ez} 0}\right)$ and is zero only at $V_{\mathrm{phs}}=V_{\mathrm{ez} 0}$. The width of the region in $V_{\text {ez0 }}$ parameter-space $\left(V_{e z}=V_{\text {ez } 0}+v_{e z}\right)$ where no periodic solutions exist is obtained from Eq. (27).

$\Delta V_{\mathrm{ez} 0}=\left(v_{e z, \max }^{-}-v_{e z, \max }^{+}\right)+2\left[\frac{\beta_{m \perp}\left(1-a_{e}\right)}{1+\tau}\right]^{1 / 2}$.

For $\beta_{m \perp}=0$ or $a_{e}=1$ (e.g. $\left.T_{e \perp} \gg T_{e \|}\right),\left(v_{e z, \max }^{-}-v_{e z, \max }^{+}\right)=0$ and thus $\Delta V_{\mathrm{ez} 0}=0$ and periodic solutions exist everywhere in the region around $V_{\mathrm{phs}}-V_{\mathrm{ez} 0}=0$.

\subsection{Linear limit}

To illustrate the connection between our model and previous work of others, we compare limiting cases of our model with related models of the time-varying, shear-mode, dispersive Alfvén wave. In the linear, or small amplitude $\left(\left|v_{e z} / V_{\mathrm{phs}}\right| \ll 1\right)$, limit, retaining electron collisions, Eq. (22) reduces to

$$
\frac{\partial^{2}}{\partial x^{2}} v_{e z}+2 \gamma \frac{\partial}{\partial x} v_{e z}+k_{x 0}^{2} v_{e z}=F
$$

The damping term $\gamma$ and the characteristic wave number $k_{x 0}^{2}$ are

$\gamma=\frac{\lambda_{e}}{2} \frac{v_{e}}{\left(V_{d} / \lambda_{e}\right)}\left[\Lambda_{e}^{2}-\frac{V_{A}^{2}}{V_{\mathrm{phs}}^{2}} \rho_{s}^{2}\left(1+\frac{V_{T i}^{2}}{C_{S}^{2}}\right)\right]^{-1}$,

and

$k_{x 0}^{2}=\left(p \frac{V_{A}^{2}}{V_{\mathrm{phs}}^{2}}-1\right)\left[\Lambda_{e}^{2} U_{e}^{2}-\frac{V_{A}^{2}}{V_{\mathrm{phs}}^{2}} \rho_{s}^{2}\left(1+\frac{V_{T i}^{2}}{C_{S}^{2}}\right)\right]^{-1}$

Equation (29) describes the response of a linear oscillator to a step-function driver (e.g., Marion and Thornton, 1995), where the forcing function $F$ is given by 


$$
\begin{aligned}
F= & U_{e}\left[V_{e z 0}+\frac{V_{A}^{2}}{V_{\mathrm{phs}}}(1-p)\right] \times \\
& {\left[\Lambda_{e}^{2} U_{e}^{2}-\frac{V_{A}^{2}}{V_{\mathrm{phs}}^{2}} \rho_{s}^{2}\left(1+\frac{V_{T i}^{2}}{C_{S}^{2}}\right)\right]^{-1} . }
\end{aligned}
$$

Here again we see that the driving term $F$ is a function of the initial currents, and is spatially homogeneous hence unstructured. In the absence of both initial currents i.e. $p=1$ and $V_{e z 0}=0$ hence $U_{e}=1$, the amplitude of the driving term is zero $F=0$ and Eq. (29) is homogeneous.

Fourier transforming Eq. (29) and substituting the relation $\tan \theta=-k_{z} / k_{x}$ from the Fourier transform of Eq. (11), for the case of strongly magnetized plasma $\omega_{c i} \rightarrow \infty$ i.e. $\Lambda_{e}^{2}=\lambda_{e}^{2}$ and no initial currents i.e. $F=0$, Eq. (29) yields

$$
-\frac{k_{x} V_{d}}{k_{z}}=V_{A} \frac{\left[1+\rho_{s}^{2} k_{x}^{2}\left(1+\frac{V_{T i}^{2}}{C_{S}^{2}}\right)\right]^{1 / 2}}{\left[1+\lambda_{e}^{2} k_{x}^{2}\left(1-i v_{e i \|} / k_{x} V_{d}\right)\right]^{1 / 2}} .
$$

For the inertial Alfvén wave in collisionless $\left(v_{e}=0\right)$, isotropic $\left(a_{e}=0\right)$ plasma, Eq. (33) reduces to the zero-frequency limit of Eq. (5) in Drozdenko and Morales (2001), describing the linear effects of cross-field plasma flow on a field-aligned current channel. Neglecting electron collisions and recognizing that $\omega=-k_{x} V_{d}$ is the effective wave frequency, Eq. (33) reduces to the dispersion relation for the low-frequency dispersive Alfvén wave including finite Larmor radius effects (Stasiewicz et al., 2000). Setting $\lambda_{e}=0$, and $\rho_{s}=0$, we see that the parallel wave phase speed is simply the Alfvén speed (Alfvén, 1942), i.e., $V_{\mathrm{phs}}=V_{A}$.

\section{Discussion}

Wave reflection at a conducting boundary is one potentially important effect in both the auroral ionosphere and a laboratory-experiment that remains unaddressed by the present model. Wave reflection of nonlinear StA waves cannot automatically be assumed to involve simple superposition, but it might. There is no obvious reason to expect that the interference produced by a reflected StA wave would inhibit the electron acceleration and plasma density variation induced by the wave, particularly since, in the linear limit, the parallel electric field associated with the inertial Alfvén wave may constructively interfere upon reflection from a conducting boundary such as the ionosphere (Goertz and Boswell, 1979).

The present StA wave model does not include background parallel and perpendicular inhomogeneities. The strong inhomogeneities present in the auroral ionosphere may make the establishment of a StA wave pattern at a fixed angle to the background magnetic field doubtful. Of particular importance is the functional dependence of the electron and ion collision frequencies with altitude. Inhomogeneities in the collision frequencies would have to be addressed before making any direct applications of the collisional StA wave to the auroral zone. Predicting the details of interference and reflection as well as parallel and perpendicular inhomogeneities may be accomplished more accurately via computer simulation.

Along auroral magnetic-field-lines, thermal effects become increasingly significant at altitudes above $2 \mathrm{R}_{E}\left(\mathrm{R}_{E}\right.$ is Earth radius). For stationary inertial Alfvén waves with warm plasma corrections (non-zero parallel electron thermal pressure), the parallel component of electric field becomes singular only when the initial electron drift is parallel to (PED solutions) and equal to the parallel wave phase velocity in the convecting plasma rest frame i.e., $V_{e z}=V_{\text {phs }}$. For initial electron drifts anti-parallel to the parallel wave phase velocity (APED solutions), the parallel component of electric field is nonsingular for all values of initial electron drift and the resulting resonant acceleration discussed both here and by Kletzing (1994) is unchanged. Thus, the altitude range of periodic StA wave structures in the auroral ionosphere may be limited for initial electron drifts which are parallel to the effective parallel wave phase velocity, as perceived in the convecting plasma rest frame.

\section{Conclusions}

In this paper, a two-fluid model describing a collisional stationary Alfvén wave in anisotropic plasma is presented. A bounded range of values of parallel electron drift velocity is found that excludes periodic stationary Alfvén wave solutions. This exclusion region depends on parameters $V_{A}, \beta_{m \perp}$ and electron temperature anisotropy.

Acknowledgements. This work is supported by a US NSF/DOE grant at WVU (PHYS-0613238). D. K. is supported by the National Research Council (Canada). This work is part of the Space-Plasma Campaign being carried out by M. Koepke, S. Finnegan, D. Knudsen, R. Rankin, R. Marchand, C. Chaston, and S. Vincena at the Basic Plasma Science Facility, University of California, Los Angeles and thus receives part of the facility support from NSF and DOE. A useful discussion with G. Ganguli who suggested that we address pressure anisotropy, is gratefully acknowledged.

Edited by: T. Chang

Reviewed by: E. J. Lund and another anonymous referee

\section{References}

Alfvén, H.: Existence of electromagnetic-hydrodynamic waves, Nature, 150, 405, 1942.

Allen, T. K., Baker, W. R., Pyle, R. V., and Wilcox, J. M.: Experimental generation of plasma Alfvén waves, Phys. Rev. Lett., 2, 383, 1959.

Chaston, C. C., Bonnell, J. W., Carlson, C. W., McFadden, J. P., Ergun, R. E., Strangeway, R. J., and Lund, E. J.: Auroral ion 
acceleration in dispersive Alfvén waves, J. Geophys. Res., 109, A04205, doi:10.1029/2003JA010053, 2004.

Chust, T., Roux, A., Kurth, W. S., Gurnett, D. A., Kivelson, M. G., and Khurana, K. K.: Are Io's Alfvén wings filamented?-Galileo observations, Planet. Space Sci., 53, 395-412, 2005.

Demars, H. G. and Schunk, R. W.: Temperature anisotropies in the terrestrial ionosphere and plamasphere, Rev. Geophys. Space Ge., 25, 1659, doi:10.1029/RG025i008p01659, 1987.

Drozdenko, T. and Morales, G. J.: Effect of cross-field flow on inertial Alfvén waves of small transverse scale, Phys. Plasmas, 8, 3177, doi:10.1063/1.1374587, 2001.

Dubinin, E., Sauer, K., and McKenzie, J. F.: Nonlinear inertial and kinetic Alfvén waves, J. Geophys. Res., 110, A10S04, doi:10.1029/2004JA010770, 2005.

Finnegan, S. M., Koepke, M. E., and Knudsen, D. J.: The dispersive Alfvén wave in the time-stationary limit with a focus on collisional and warm-plasma effects, Phys. Plasmas, 15, 052108, doi:10.1063/1.2890774, 2008.

Gekelman, W., Vincena, S., Leneman, D., and Maggs, J.: Laboratory Experiments on Shear Alfvén Waves and Their Relationship to Space Plasmas, J. Geophys. Res., 102, 7225, doi:10.1029/96JA03683, 1997.

Goertz, C. K. and Boswell, R. W.: MagnetosphereIonosphere coupling, J. Geophys. Res., 84, 7239, doi:10.1029/JA084iA12p07239, 1979.

Hasegawa, A. and Mima, K.: Exact solitary Alfvén wave, Phys. Rev. Lett., 37, 690, doi:10.1103/PhysRevLett.37.690, 1976.

Hasegawa, A. and Uberoi, C.: The Alfvén Wave, DOE Critical Review Series-Advances in Fusion Sciences and Engineering, Technical Information Center, US Dept. of Energy, 1982.

Hui, C. H. and Seyler, C. E.: Electron acceleration by Alfvén waves in the magnetosphere, J. Geophys. Res., 97, doi:10.1029/91Ja03101, 3953-3963, 1992.

Jephcott, D. F.: Alfvén waves in a gas discharge, Nature, 183, 1653, doi:10.1038/1831652a0, 1959.

Jephcott, D. F. and Stocker, P. M.: Hydrodynamic waves in a cylindrical plasma: An experiment, J. Fluid Mech., 13, 587, doi:10.1017/S0022112062000956, 1962.

Kletzing, C. A.: Electron acceleration by kinetic Alfvén waves, J. Geophys. Res., 99, A6, 11 095-11 104, doi:10.1029/94JA00345, 1994.

Knudsen, D. J.: Spatial modulation of electron energy and density by nonlinear stationary inertial Alfvén waves, J. Geophys. Res., 101, A5, 10 761-10 772, doi:10.1029/96JA00429, 1996.

Koepke, M. E., Finnegan, S. M., Vincena, S., Knudsen, D. J., and Chaston, C.: Integrated campaign to study the stationary inertial Alfvén wave in the laboratory and space regimes, Plasma Phys. Contr. F., 50, 074004, doi:10.1088/0741-3335/50/7074004, 2008.

Kotsarenko, N. Y., Rapoport, Y. G., Shvidkij, A. A., and Khotyaintsev, Y. V.: Oblique Alfvén and magnetosonic solitons and nonlinear structures in magnetized plasmas, Phys. Scripta, 58, 499, doi:10.1088/0031-8949/58/5/013, 1998.

Leneman, D., Gekelman, W., and Maggs, J.: Laboratory Observations of Shear Alfvén Waves Launched from a Small Source, Phys. Rev. Lett., 82, 2673, doi:10.1103/PhysRevLett.82.2673, 1999.
Lysak, R. L. and Lotko, W.: On the kinetic dispersion relation for shear Alfvén waves, J. Geophys. Res., 101, 5085, doi:10.1029/95JA03712, 1996.

Lysak, R. L. and Song, Y.: Kinetic theory of Alfvén wave acceleration of auroral electrons, J. Geophys. Res., 108, 8005, doi:10.1029/2002JA009406, 2003.

Mallinckrodt, A. J. and Carlson, C. W.: Relations between transverse electric fields and field-aligned currents, J. Geophys. Res., 83, 1426, doi:10.1029/JA083iA04p01426, 1978.

Maltsev, Y. P., Lyatsky, W. B., and Lyatskaya, A. M.: Currents over the auroral arc, Planet. Space Sci., 25, 53, doi:10.1016/00320633(77)90117-9, 1977.

Marion, J. B. and Thornton, S. T.: Classical Dynamics of Particles and Systems, Fourth Edition, Harcourt Brace and Company, 140-143, 1995.

Oyama, K.-I. and Takumi, A.: Anisotropy of electron temperature in the ionosphere, Geophys. Res. Lett., 14, 1195, doi:10.1029/GL014i012p01195,1987.

Rankin, R., Samson, J. C., and Tikhonchuk, V. T.: Parallel electric fields in dispersive shear Alfvén waves in the dipolar magnetosphere, Geophys. Res. Lett., 26, 3601, doi:10.1029/1999GL010715, 1999.

Sallago, P. A. and Platzeck, A. M.: Alfvén waves and wings in Hall magnetohydrodynamics, J. Geophys. Res., 109, A04218, doi:10.1029/2003JA009920, 2004.

Seyler, C. E. and Liu, K.: Particle energization by oblique inertial Alfvén waves in the auroral region, J. Geophys. Res., 112, A09302, doi:10.1029/2007JA012412, 2007.

Seyler, C. E., Wahlund, J. E., and Holback, B.: Theory and simulation of low-frequency plasma waves and comparison to Freja satellite observations, J. Geophys. Res., 100, 21 453-21472, doi:10.1029/95JA02052, 1995.

Stasiewicz, K.: Nonlinear Alfvén, magnetosonic, sound, and electron inertial waves in a fluid formalism, J. Geophys. Res., 110, A03220, doi:10.1029/2004JA010852, 2005.

Stasiewicz, K., Bellan, P., Chaston, C., et al.: Small Scale Alfvénic Structure in the Aurora, Space Sci. Rev., 92, 423, doi:10.1023/A:1005207202143, 2000.

Streltsov, A. and Lotko, W.: Dispersive field line resonances on auroral field lines, J. Geophys. Res., 100, 19457, doi:10.1029/95JA01553, 1995.

Temerin, M., McFadden, J., Boehm, M., Carlson, C. W., and Lotko, W.: Production of flickering aurora and field-aligned electron flux by electromagnetic ion cyclotron waves, J. Geophys. Res., 91, 5769, doi:10.1029/JA09liA05p05769, 1986.

Vincena, S., Gekelman, W., and Maggs, J.: Shear Alfvén Wave Perpendicular Propagation from the Kinetic to the Inertial Regime, Phys. Rev. Lett., 93, 105003, doi:10.1103/PhysRevLett.93.105003, 2004.

Wilcox, J. M., Boley, F. I., and DeSilva, A. W.: Experimental study of Alfvén wave properties, Phys. Fluids, 3, 15, doi:10.1063/1.1705996, 1960.

Wu, D. J.: Dissipative solitary kinetic Alfvén wave and electron acceleration, Phys. Plasmas, 10, 1364, doi:10.1063/1.564821, 2003. 